\title{
Regional symmetry of the pelvis
}

Sarah McClelland ${ }^{1}$, Samantha Polege ${ }^{1}$, David Li ${ }^{1}$, Maha Ead ${ }^{1}$, Lindsey Westover ${ }^{1}$, Kajsa Duke ${ }^{1}$

${ }^{1}$ Department of Mechanical Engineering, University of Alberta

\begin{abstract}
Understanding the left-right symmetry of the pelvis can assist with the virtual reconstruction of fractured pelvises in the future. Further specifying the different regions can give more information regarding the anatomy of the bone. CT scans of intact pelvises are turned into 3D models using a computer program called Mimics. The femurs and spine are removed manually to isolate the bone. The model can then be analyzed and the regions defined. The pelvis is split into three pieces using the program Geomagic, the iliac, acetabular, and pubic rami regions. The two cuts are made based around the highest and lowest points of the acetabular cup. From here each cut piece can be mirrored and aligned with the opposing side of the pelvis. Colour deviation maps can then be generated to visualize the asymmetry, as well as the RMS value and the percentage of points within a $2 \mathrm{~mm}$ deviation threshold. The average RMS values are below $2 \mathrm{~mm}$ and the percentage of points within $2 \mathrm{~mm}$ is high. My research reflects that the pelvis is symmetrical and may be used to assist in the surgical planning process of pelvic fractures.
\end{abstract}

Key words:

Pelvis, symmetry, pelvic fracture, left-right symmetry, virtual reconstruction, lliac, Pubic Ramus, Pubic Rami, Acetabulum

Cite as: McClelland, S., Polege, S., Li, D., Ead, M., Westover, L., Duke, K. 2019. Regional Symmetry of the Pelvis. Alberta Academic Review, Vol 2 (2) 53-54, WISEST Special Issue (non peer-reviewed), DOI 10.29173/aar62. 
McClelland et al., 2019

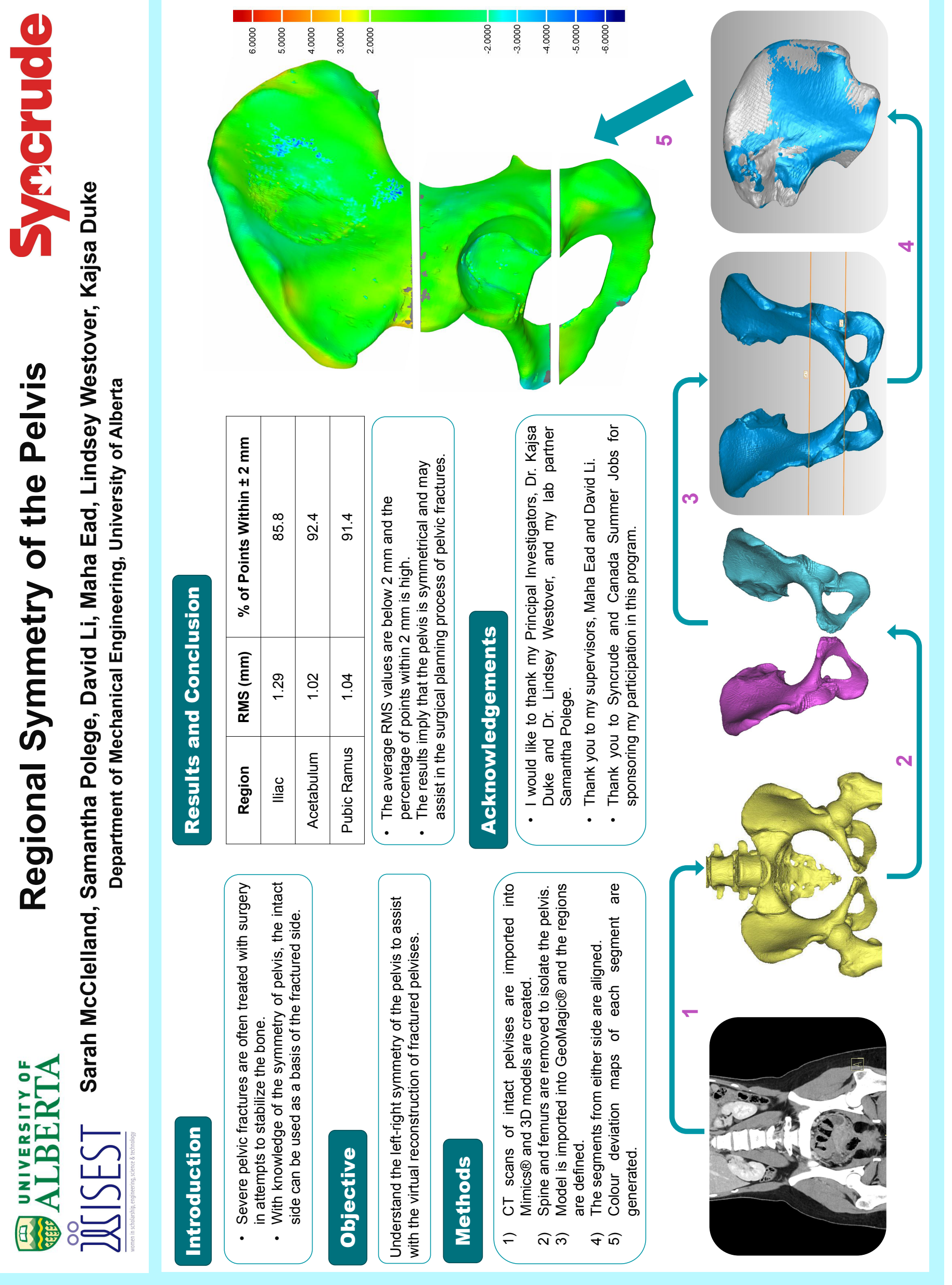

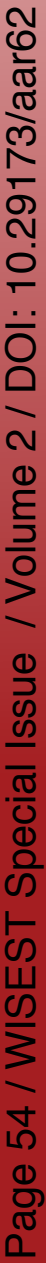

